\title{
Epidemiology and antibiotic resistance in community-acquired lower urinary tract infections in the Milan area
}

\author{
Daniele Paris, Mariasofia Caltagirone, Patrizia Minzulli, Antonia Valzano, Fulvio Enrico Ottorino Ferrara, \\ Enrico Magliano
}

Centro Diagnostico Italiano, Milan, Italy

\begin{abstract}
Summary
Urinary tract infections (UTIs) are among the most common bacterial infectious diseases occurring in the community and healthcare setting. Most community-acquired urinary tract infections are usually treated empirically. The knowledge of antibiotic resistance patterns of the microorganisms causing UTI is essential for defining the empirical treatment. The aim of the present study is to investigate the prevalence and the resistance patterns of bacterial species isolated from lower urinary tract infections, in a large population of Milan area. A retrospective analysis of the isolates obtained from urine samples received at the microbiology laboratory of Centro Diagnostico Italiano of Milan was performed from January 2019 to December 2019. Urine samples were plated on differential medium by automated inoculation system. Identification and antibiotic susceptibility testing were performed using the Phoenix $100^{\mathrm{TM}}$ system. All results were interpreted according to the European Committee on Antimicrobial Susceptibility Testing breakpoints. During a 12-month period a total
\end{abstract}

Correspondence: Fulvio Enrico Ottorino Ferrara, Via Saint Bon 20, 20147 Milano, Italy.

Tel: $+39.02 / 48317499$.

E-mail: fulvio.ferrara@cdi.it

Key words: Urinary tract infection, community-acquired infection, antibiotic resistance.

Contributions: The authors contributed equally.

Conflict of interest: The authors declare no potential conflict of interest.

Funding: None.

Availability of data and materials: Data and materials are available upon request from the corresponding author.

Received for publication: 9 June 2020.

Accepted for publication: 21 July 2020.

${ }^{\circ}$ Copyright: the Author(s), 2020

Licensee PAGEPress, Italy

Microbiologia Medica 2020; 35:9269

doi:10.4081/mm.2020.9269

This article is distributed under the terms of the Creative Commons Attribution Noncommercial License (by-nc 4.0) which permits any noncommercial use, distribution, and reproduction in any medium, provided the original author(s) and source are credited. of the 51,980 urine samples have been processed and 21,4\% (11.148) were found to be positive (bacterial count $\geq 10^{5} \mathrm{CFU} / \mathrm{mL}$ ). Overall Escherichia coli was the most common Gram-negative bacteria of all isolates (72\%), followed by Klebsiella pneumoniae (10.8\%) and Proteus mirabilis (3.3\%). Susceptibility of E. coli to oral antimicrobial agents was demonstrated to be as follows: fosfomycin (97\%), trimethoprim/sulfamethoxazole $(76 \%)$, ciprofloxacin (89\%), ampicillin (51\%) and amoxicillin/clavulanate $(77 \%)$. The present study point-out the common antibiotic resistance trend of uropathogens in this area. Our results will help in the formulation of antibiotic policy and determination of empirical treatment of urinary tract infection.

\section{Introduction}

Urinary tract infection (UTI) are among the most frequent bacterial infections world-wide (1).

Uncomplicated UTIs typically occur in the healthy premenopausal, nonpregnant women with no history suggestive of an abnormal urinary tract. Complicated UTIs may occur in both women and men and in any age groups and are frequently associated with either structural or functional urinary tract abnormalities. Most infections occur in the lower urinary tract. The microbial spectrum of UTIs consists mainly of E. coli, Klebsiella spp., Proteus spp., Enterococcus spp., Enterobacter spp. and Staphylococcus saprophyticus the commonest urinary pathogen in young women $(1,2)$.

The use of an initial appropriate antibiotic usually improves a patient's prognosis. Earlier studies have reported that the indiscriminate use of antibiotics for treating UTIs resulted in a lower cure rate, longer hospital stay, higher relapse rate and accelerated development of antibiotic resistance (3).

UTIs caused by antibiotic-resistant Gram-negative bacteria are a growing concern due to limited therapeutic options. Gramnegative bacteria, specifically Enterobacterales, are common causes of both community-acquired and hospital acquired UTIs. These organisms can acquire genes that encode for multiple antibiotic resistance mechanisms, including AmpC- $\beta$-lactamase, extended-spectrum-lactamases (ES $\beta$ Ls) and carbapenemases (4).

The most recent data from the European Antimicrobial Resistance Surveillance Network (EARS-Net) reported in Italy very high resistance proportions to extended-spectrum cephalosporins among invasive bacterial pathogens: E. coli $(28.7 \%)$ and $K$. pneumoniae (53.6\%) (5).

Moreover, the Italian nationwide survey carried out in 2013 showed a notable increase of ES $\beta$ L-producing Enterobacteriaceae $(11.0 \%)$ and the presence of KPC-producing K. pneumoniae $(4.6 \%)$ 
from outpatients $(6)$

The aim of the present study is to investigate the prevalence of bacterial species isolated from lower urinary tract infections and their resistance patterns against commonly used antimicrobial agents, in a large population of Milan area.

\section{Materials and Methods}

In the Microbiology Laboratory of the "Centro Diagnostico Italiano" (CDI) of Milan, certificated Joint Commission International (JCI) was performed a retrospective study on all bacterial strains isolated from consecutive urine samples, received from outpatients of a high-populated urban area of North Italy between January 2019 and December 2019.

CDI Laboratory follows Internal Quality Control procedures and participates to an External Program for Quality Assessment with positive evaluations.

Patients received indications to avoid antimicrobials assumption during the previousdays and instructions on urine sampling (including cleaning of the genital area prior to midstream specimen collection) and its transport to the laboratories (within 2 hours of collection). Specimens from external laboratories were transported in Vacutainer tubes containing boric acid at 1-2\% as preservative.

Urine samples were plated, as soon as possible (two hours) on Chromagar Orientation medium (BD Diagnostics, MD) by automated inoculation system Walk-Away Specimen Processor (WASP) (Copan, Italy) with a $10-\mu$ loop. The inoculated chromogenic agar plates were incubated for $18-24$ hours at $37^{\circ} \mathrm{C}$, as recommended by the manufacturer.

Criterion for defining significative bacteriuria was the presence of $\geq 10^{5}$ colony forming units $/ \mathrm{mL}$ urine.

Identification and antibiotic susceptibility testing were performed using the Phoenix 100 ${ }^{\mathrm{TM}}$ system (BD Dickinson and Company, Franklin Lakes, NJ). Phoneix AST antimicrobials panels for Gram-negative urinary pathogens, Gram-negative nonurinary pathogens, Streptococci and all other Gram-positive were used. The results were interpreted according to EUCAST guidelines 2020 (7).

\section{Results}

A total of 51,980 urine cultures were performed over a 12 months period; $34,716(68 \%)$ of all were from females and 16,536 $(32 \%)$ from males (Figure 1).

A total of 11,148 (21,45\%) samples showed significant growth ( $\geq 10^{5}$ colony-forming units) after incubation; $9,141(82 \%$ ) of all isolates were from females and 2007 (18\%) from males (Figure 2).

Gram-negative agents represented $93.1 \%$ of urinary pathogens. Overall the most frequently encountered pathogen was $E$. coli (72\%), followed by K. pneumoniae (10.9\%), E. faecalis (4.7\%), P. mirabilis (3.4\%), Pseudomonas aeruginosa (1.8\%), Citrobacter koseri $(1.5 \%)$, Enterobacter cloacae $(1.1 \%)$, K. oxytoca $(1 \%)$ and others $(3,6 \%)$ (Figure 3$)$.

Frequency of isolation of the main species was found to be different between females and males. All species were more frequent in females, whereas $S$. aureus and P. aeruginosa were more common in men. The species A. baumannii shows the same frequency in both genders (Figure 4).

Susceptibility to antimicrobials of main isolated uropathogens is shown in Table 1.

E. coli susceptibility to orally active compounds ranged from
$51.0 \%$ (ampicillin) to $98.0 \%$ (nitrofurantoin). Tested quinolone compound (ciprofloxacin) resulted to be active against $75 \%$ of uropathogenic E. coli comparable to amoxicillin/clavulanate activity (77\%).

Susceptibility of $E$. coli to cephalosporins ranged from $82 \%$ to 92\% while rates for carbapenems (meropenem; imipenem), aminoglycosides (gentamicin; tobramicin) and piperacillin/ tazobactam was higher (from $91 \%$ to $99 \%$ ). The resistance rate of $E$. coli to cotrimoxazole was $3 \%$.

K. pneumoniae resistance to ampicillin (99\%) was higher in comparison to $E$. coli, susceptibility of to cephalosporins ranged from $81.5 \%$ to $85.3 \%$ and to carbapenems from $95.5 \%$ to $97.2 \%$, while

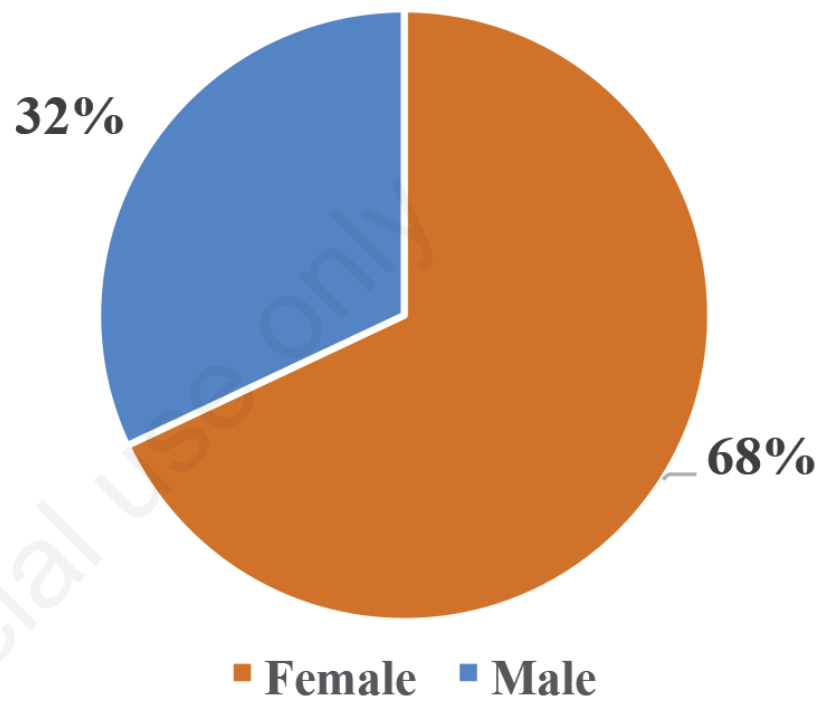

Figure 1. Gender distribution of 51980 urine samples.
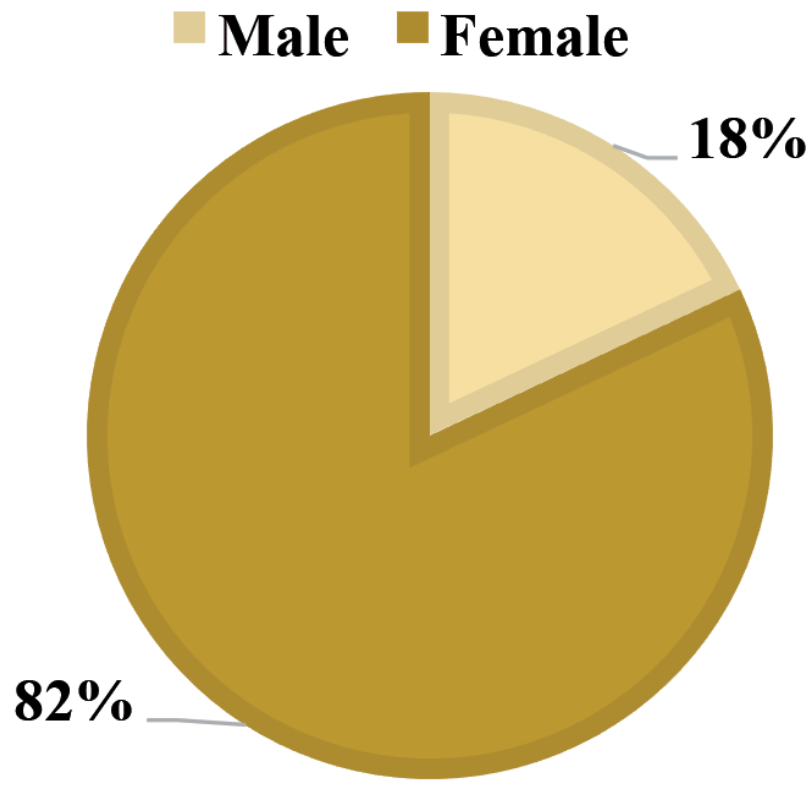

Figure 2. Distribution of positive cultures according to patients' gender. 
the ciprofloxacin resulted to be active against $80 \%$ of isolates. Cotrimoxazole exhibited high activity against $K$. pneumoniae $(86.8 \%)$.

Susceptibility of $P$. mirabilis to ciprofloxacin resulted to be lower in comparison to E. coli and K. pneumoniae, while the cephalosporins resulted to be active with a resistance that ranged from $4.6 \%$ to $9.2 \%$.

E. faecalis showed high level of susceptibility to ciprofloxacin (80\%) and aminoglycosides (gentamicin 94\%; tobramicin 89\%).

All classes of antimicrobials tested against $P$. aeruginosa resulted to be active ranged from $85.4 \%$ (gentamicin) to $97.3 \%$ (meropenem).

\section{Discussion and Conclusions}

Urinary tract infections (UTIs) both in males and females represent one of the major cause of urological consultation in our clinical practice.

Half of all women will have at least one episode of acute cystitis during their adult life and one-quarter will also report recurrent episode (8).

The UTI refers to the presence of a certain number of bacteria in the urine (generally $>10^{5} / \mathrm{ml}$ ) and symptomatic UTIs are classified in order of severity as urosepsis syndrome, pyelonephritis

Table 1. Susceptibility rates to antimicrobials of most common uropathogens isolated from urine samples.

\begin{tabular}{lcccccccccccccccccc} 
Organism(n; \%) & CIP & TGC & NIT & SXI & AMP & AMC & TZP & CAZ & CIX & FEP & MRP & IMP & GEN & TM & THC & LAD & VA \\
E. coli $(8027,72 \%)$ & 75 & 94.8 & 98 & 76 & 51 & 77 & 97 & 92 & 85 & 89 & 99 & 99 & 92 & 91 & 99.4 & 99.6 & 99.6 & \\
K. pneumoniae $(1.212,10.9 \%)$ & 80 & NT & NT & 83.8 & 1 & 75.8 & 86.8 & 85.3 & 85.6 & 81.5 & 95.5 & 97.2 & 93.2 & 89.3 & NT & NT & NT \\
\hline E. faecalis $(527,4.73 \%)$ & 80.9 & 99.8 & 98.7 & NT & 99.8 & NT & NT & NT & NT & NT & NT & 99.6 & NT & NT & NT & NT & NT \\
P. mirabilis $(377,3.38 \%)$ & 59.8 & NT & NT & 61 & 48.2 & 83.4 & 98.3 & 93.9 & 90.8 & 95.4 & 100 & NT & 40.1 & 69.4 & NT & NT & NT \\
\hline P. aeruginosa $(199,1.79 \%)$ & 77.5 & NT & NT & NT & NT & NT & 90.7 & 87.8 & NT & 87.7 & 97.3 & 95.1 & 85.4 & 90.7 & NT & NT & NT
\end{tabular}

Data are presented as percentage; NT: not tested; CIP: ciprofloxacin; TGC: tigecycline; FOS: fosfomycin; NIT: nitrofurantoin; SXT: trimethoprim-sulfamethoxazole; AMP: ampicillin; AMC: amoxicillin/clavulanic acid; TZP: piperacillin/tazobactam; CAZ: ceftazidime; CTX: cefotazime; FEP: cefepime; MRP: meropenem; IMP: imipenem; GEN: gentamicin; TM: tobramicin; TEC: teiocoplanin, LZD: linezolid; VA: vancomycin.

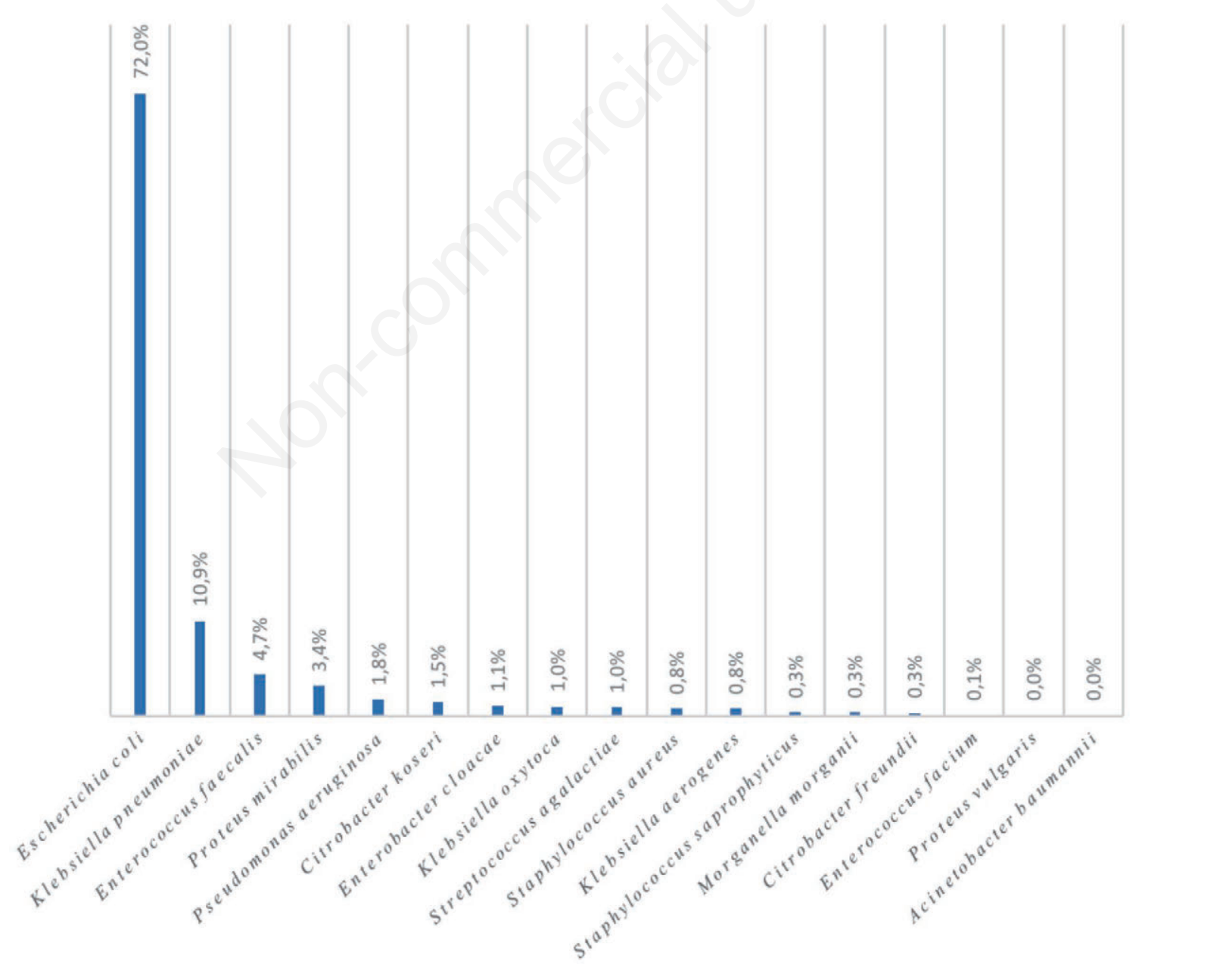

Figure 3. Distribution of bacterial isolates species from urine samples $(\mathrm{n}=11,148)$. 
(or upper UTI, with infection in the kidney) and cystitis (or lower UTI, with bacteria into the bladder;). Clinically, UTIs classification comprises either uncomplicated or complicated cases, depending on the presence of structural or neurological urinary tract abnormalities (9).

International guidelines for the treatment of uncomplicated UTIs and pyelonephritis recommend various agents, such as nitrofurantoin monohydrate, trimethoprim-sulfamethoxazole, fosfomycin trometamol, fluoroquinolones and beta-lactams (10).

In Community, irrational drug use, such as low dose antibiotics, long-term use and empiric therapy are usually reporter (11).

Appropriate empirical therapy is important since treatment with an antimicrobial to which the uropathogen is resistant is associated with more clinical and microbiological failures: a longer median time to symptom resolution, higher re-consultation rates and more subsequent antibiotics.

When deciding on empiric therapy for a UTI, local resistance patterns to antibiotics are an important factor in choice of therapy. Given this consideration, antibiotic choice should be based not only on efficacy and safety, but also, on the concept that broad spectrum antibiotics should be spared in order to safeguard their future effectiveness.

The study of Ussai et al. clearly demonstrates that Italian primary-care physicians don't follow best practices when empirically managing UTI's and often change their prescribing patterns during therapy, moreover the authors reported specifically a quinolones overuse among Italian outpatients, even if according to the European Urology Guidelines for UTIs, neither quinolones nor cephalosporins are appropriate for the empiric treatment of uncomplicated cystitis (12).

The antibiotic overuse raises concern about resistance to a particular UTI drug or class of antibiotics. In particular, the progressive increase in resistance to broad-spectrum antibiotics, such as third-generation cephalosporins, fluoroquinolones or carbapenems in Enterobacterales, is an alarming situation for all urologists and general practitioners.

The availability of published surveillance data on antimicrobial resistance in uropathogens in community-acquired UTI is limited, moreover the European Antimicrobial Resistance Surveillance System reports resistance frequencies in invasive isolates but does not report data on resistance surveillance in community-acquired UTI, a much more common clinical condition (13).

For this reason, the aim of the present study is to determine the prevalence of bacterial species isolated from lower urinary tract infections and their resistance patterns against commonly used antimicrobial agents.

The present retrospective study describes the distribution and antimicrobial susceptibility of bacterial species isolated from a large number of urinary samples collected over a 12-month period, from patients of northern Italy.

Results pointed to a higher incidence of urinary tract infections in women than men. This is primarily due to differences in the urogenital system. In fact, women have much shorter urethra than men, and its opening is much closer to the outlet of colon and vagina, where it can be more easily contaminated by pathogenic mucosa and cause an infection (14).

Our results confirm that $E$. coli was the most frequently encountered species causing community-acquired UTIs. Other frequent isolates found in this study included $K$. pneumoniae, $P$. mirabilis, and E. faecalis, all having been reported to be highly prevalent species in UTIs and $P$. aeruginosa.

Among the antimicrobial compounds tested in our study trimethoprim-sulfamethoxazole and nitrofurantoin exhibited the highest activity against E. coli $(97 \% ; 98 \%)$.

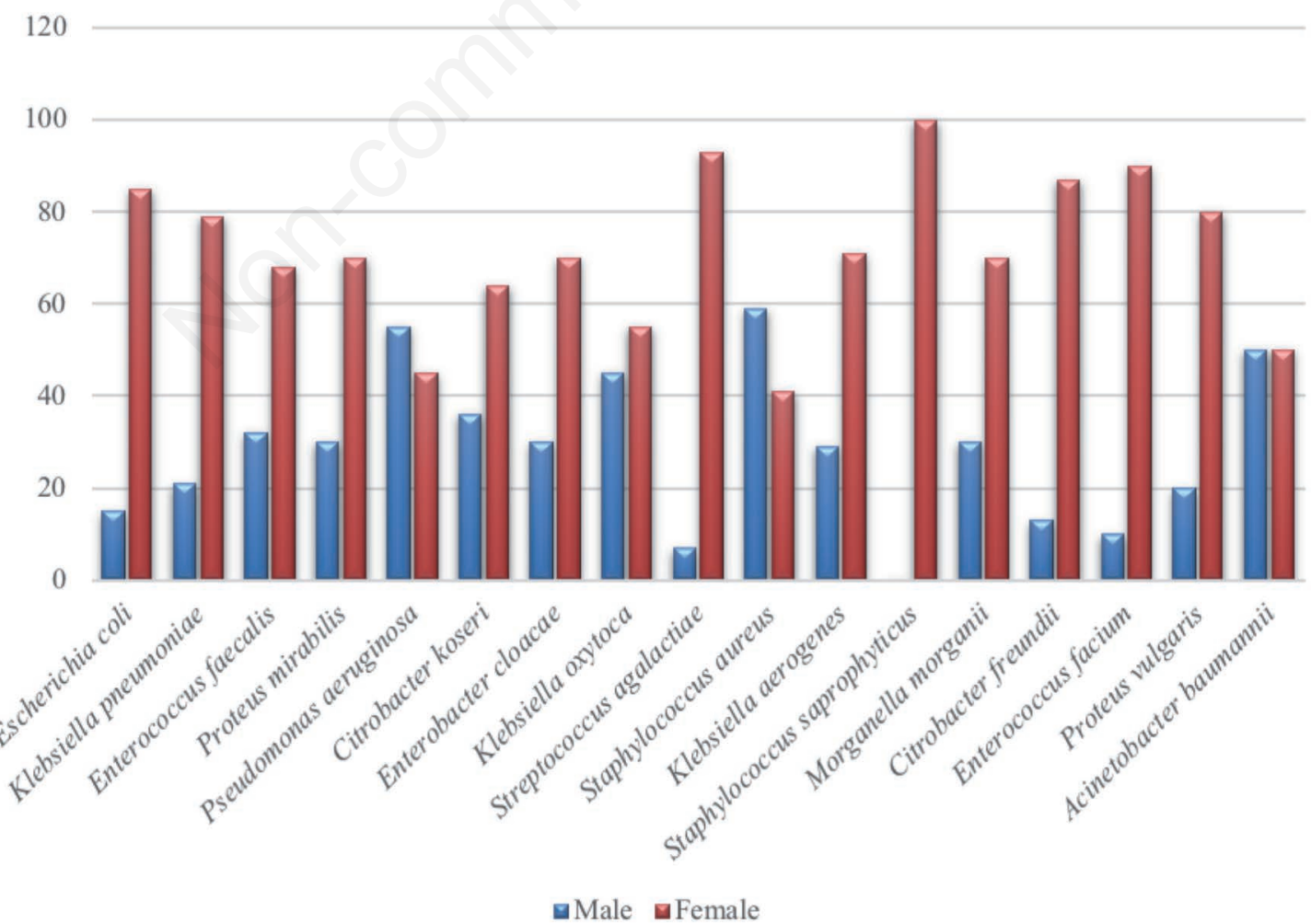

Figure 4. Distribution of bacterial species according to patients' gender. Data are reported as percentages. 
Table 2. Proportions of ESCR-carbaS and ESCR-carbaR of Enterobacteriaceae: comparison between the present study and nationwide surveillance survey (Italy, October 2013).

\begin{tabular}{lccccccccccc} 
Species & \multicolumn{3}{c}{ Isolates from inpatients (CDI) } & \multicolumn{5}{c}{ Isolates from inpatients (Italian survey) } \\
& Total & ESCR-Carba S & $\%$ & ESCR-Carba R & $\%$ & Total ESCR-Carba S & $\%$ & ESCR-Carba R & \% \\
E. coli & 8027 & 883 & 11 & 8 & 0.1 & 1,432 & 159 & 11.1 & 3 \\
K. pneumoniae & 1.212 & 228 & 18.5 & 34 & 2.8 & 260 & 16 & 6.2 & 20 \\
\hline P. mirabilis & 377 & 17.3 & 4.6 & 0 & 0 & 123 & 26 & 21.1 & 0 \\
\hline
\end{tabular}

ESCR-carbaS: non-susceptible to extended-spectrum cephalosporins but susceptible to carbapenems; ESCR-carbaR: isolates non-susceptible to extended-spectrum cephalosporins and non-susceptible to carbapenems.

Trimethoprim-sulfamethoxazole was a typical antibiotic used to treat UTIs, authors emphasized its role of this antibiotic in empirical antibiotics because of the recent decrease in the resistance rate in several European countries due to its low prescription rate (15).

In our survey, E. coli susceptibility to ampicillin was found to be low (51\%) while higher to amoxicillin/clavulanate $(77 \%)$, comparable to the previous report $(48 \% ; 77.5 \%)$ conducted by Magliano E. et al., between March 2008 and December 2009 in the same urban area of north of Italy (16). High level of ampicillin resistance could be due to plasmid-mediated transmission of genes encoding $\beta$-lactamases.

Tested quinolone compound (ciprofloxacin) resulted to be active against $75 \%$ of uropathogenic $E$. coli which was similar to rates observed in Italy by Magliano et al. (76\%) (16).

$K$. pneumoniae resistance to ampicillin (99\%) was higher in comparison to E. coli $(33.9 \%)$, this phenotype can be related to production of the chromosomal $\beta$-lactamase (e.g., SHV) (15).

Susceptibility of $P$. mirabilis to ciprofloxacin and trimethoprimsulfamethoxazole resulted to be lower $(59.8 \% ; 61 \%)$ in comparison to $E$. coli and K. pneumoniae.

The percentage of Gram-negative isolates confirmed to be non-susceptible to cephalosporins was comparable to the data of Italian nationwide surveillance carried out in 2013 (6): $E$. coli $(11 \%$ vs $11.1 \%) K$. pneumoniae $(18.5 \%$ vs 6.2$)$ and $P$. mirabilis $(4.6 \%$ vs $21.1 \%)$ (Table 2$)$. The proportion of carbapenem resistant $K$. pneumoniae isolates was lower in comparison to the data of Italian survey $(2.8 \%$ vs $7.7 \%)$, moreover this phenotype was found to be relatively common in E. coli and $P$. mirabilis (Table 2).

In this study, we didn't use the reference method agar dilution for testing susceptibilities to fosfomycin, this is a limit of our study in view of this antibiotic is often used for the treatment of uncomplicated urinary tract infections as a single-dose oral form.

Furthermore, in the case of beta-lactams resistant bacterial strains, the enzymatic class involved has not been determined by phenotypic or molecular tests, but this will be the target of our next study.

The etiology of bacteria causing UTI as well as their susceptibility to antimicrobials continue to vary over time period and it is different among different countries. The Infectious Diseases Society of America recommends that regional surveillance should be conducted to monitor changes in susceptibility of uropathogens in specific regions (17).

Surveillance of local UTI's etiology as well as of antimicrobial susceptibility is considered useful to guide empirical therapy, as prevalence of pathogens and their features may vary with time and geographical area.

These data provide additional information about the type of pathogens causing UTIs and their antimicrobial susceptibility patterns and can be used as a basis to develop national countryspecific guidelines for the empirical treatment of UTIs.

\section{References}

1. Kothari A, Sagar V. Antibiotic resistance in pathogens causing community-acquired urinary tract infections in India: a multicenter study. J Infect Dev Ctries 2018;2:354-8.

2. Erdem I, Kara Ali R, et al. Community-acquired Lower Urinary Tract Infections: Etiology, Antimicrobial Resistance, and Treatment Results in Female Patients. J Glob Infect Dis 2018;10:129-32.

3. Kim YJ, Lee JM, Cho J, Lee J. Change in the Annual Antibiotic Susceptibility of Escherichia coli in Community-Onset Urinary Tract Infection between 2008 and 2017 in a Tertiary Care Hospital in Korea. J Korean Med Sci 2019;34:228.

4. Bader MS. An update on the management of urinary tract infections in the era of antimicrobial resistance. Postgrad Med 2017; 129:242-58.

5. European Center for Disease Prevention and Control (ECDC). Antimicrobial resistance interactive database (EARS-Net).

6. Giani T, Antonelli A, Caltagirone M, et al. Evolving betalactamase epidemiology in Enterobacteriaceae from Italian nationwide surveillance, October 2013: KPC-carbapenemase spreading among outpatients [published correction appears in Euro Surveill. 2017 Aug 24;22(34):]. Euro Surveillance 2017;22:30583.

7. The European Committee on Antimicrobial Susceptibility Testing. Breakpoint tables for interpretation of MICs and zone diameters. Version 10.0, 2020. http://www.eucast.org."

8. Galatti L, Sessa A, Mazzaglia G, et al. Antibiotic prescribing for acute and recurrent cystitis in primary care: a 4 year descriptive study. J Antimicrob Chemoter 2006, 57:551-6.

9. Terlizzi ME, Gribaudo G, Maffei ME. UroPathogenic Escherichia coli (UPEC) Infections: Virulence Factors, Bladder Responses, Antibiotic, and Non-antibiotic Antimicrobial Strategies. Front Microbiol 2017;8:1566.

10. Mazzario A, Bazaj A, Cornaglia G. Multi-drug-resistant Gramnegative Bacteria Causing Urinary Tract Infections: A Review. J Chemother 2017;29:2-9.

11. Mancini A, Pucciarelli S, Lombardi FE, et al. Differences Between Community - And Hospital - Acquired Urinary Tract Infections in a Tertiary Care Hospital. New Microbiologica 2020;43:17-21.

12. Ussai S, Rizzo M, Liguori G, et al. Antibiotic Treatment of Urinary Tract Infections (UTIs) In Primary Care: An Italian Pilot Study. J Pharmacovigilance 2016;4:215.

13. Chin TL, McNulty C, Beck C, MacGowan A. Antimicrobial resistance surveillance in urinary tract infections in primary care. J Antimicrob Chemother 2016;71:2723-8.

14. Cai, T, De Nunzio, C, Salonia A, et al. Urological Infections Due to Multidrug-Resistant Bacteria: What We Need to Know? Urologia J 2016;83:21-6.

15. Lee DS, Lee SJ, Choe HS. Community-Acquired Urinary Tract 
Infection by Escherichia coli in the Era of Antibiotic Resistance. BioMed Research International. 2018

16. Magliano E, Grazioli V, Deflorio L, et al. Gender and agedependent etiology of community-acquired urinary tract infections. Sci World J 2012.

17. Ahmed SS, Shariq A, Alsalloom AA, et al. Uropathogens and their antimicrobial resistance patterns: Relationship with urinary tract infections. Int J Health Sci (Qassim) 2019;13:48-55

18. Miragliotta G,Di Pierro MN, Miragliotta L, Mosca A. Antimicrobial resistance among uropathogens responsible for community-acquired urinary tract infection in an Italian community. J Chemother 2008;20:721-7. 